\title{
Spaser and optical amplification conditions in graphene-coated active wires
}

\author{
Leila Prelat,${ }^{1}$ (1) Mauro Cuevas, ${ }^{2}$ Nicolás Passarelli, ${ }^{3}$ Raúl Bustos Marún ${ }_{9}^{4}$ and \\ Ricardo Depine ${ }^{1, *}$
}

\begin{abstract}
${ }^{1}$ Grupo de Electromagnetismo Aplicado, Departamento de Física, Universidad de Buenos Aires and IFIBA, Consejo Nacional de Investigaciones Científicas y Técnicas, Ciudad Universitaria, Pabellón I, Buenos Aires 1428, Argentina

${ }^{2}$ Consejo Nacional de Investigaciones Científicas y Técnicas and Facultad de Ingeniería, Universidad Austral, Pilar, Buenos Aires 1629, Argentina ${ }^{3}$ Instituto de Ciencias de Materiales de Barcelona, Consejo Nacional de Investigaciones Científicas, 08193 Barcelona, Spain

${ }^{4}$ Instituto de Física Enrique Gaviola, Consejo Nacional de Investigaciones Científicas y Técnicas and Facultad de Ciencias Químicas, Universidad Nacional de Córdoba, Ciudad Universitaria, Córdoba 5000, Argentina

*Corresponding author: rdep@df.uba.ar
\end{abstract}

Received 1 March 2021; revised 6 May 2021; accepted 26 May 2021; posted 27 May 2021 (Doc. ID 423734); published 18 June 2021

\begin{abstract}
This work analyzes the optical properties of a localized surface plasmon (LSP) spaser made of a dielectric active wire coated with a graphene monolayer. Our theoretical results, obtained by using rigorous electromagnetic methods, illustrate the non-radiative transfer between the active medium and the LSPs of graphene. In particular, we focus on the lasing conditions and the tunability of the LSP spaser in two cases: when the wire is made of an infrared/terahertz transparent dielectric material and when it is made of a metal-like material. We analyze the results by comparing them with analytical expressions obtained by using the quasistatic approximation. We show that the studied systems present a high tunability of the spaser resonances with the geometrical parameters as well as with the chemical potential of graphene. (0)2021 Optical Society of America
\end{abstract}

https://doi.org/10.1364/JOSAB.423734

\section{INTRODUCTION}

Spaser, which stands for surface plasmon amplification by stimulated emission of radiation, is the counterpart of a laser that ideally emits surface plasmons instead of photons. Due to the coupling of localized surface plasmons (LSPs) with electromagnetic radiation, a spaser can confine light at subwavelength scales, which can be used to provide a controllable source of on-demand high-intensity electromagnetic fields beyond the diffraction limit. Since its proposal in 2003 [1] and its first, to the best of our knowledge, experimental demonstrations in 2009[2], a wide variety of geometries and materials/metamaterials for the cavities as well as different compounds for the optical-gain medium have been considered [3-13]. Despite the development of many advanced spasers, most of the research in this area has been focused on natural plasmonic materials such as metals, which have a number of shortcomings, most notably power losses and a fixed charge density that finds applications in the visible and near-infrared range, but not in the mid-infrared and terahertz $(\mathrm{THz})$ region. However, under adequate circumstances, graphene and other $2 \mathrm{D}$ materials also have the ability to guide surface plasmons while having interesting advantages, such as significantly lower losses and much better tunability. In 2D materials, the constitutive parameters responsible for sustaining plasmon oscillations can be tuned with different techniques, including chemical doping and field effects. Besides, highly doped graphene shows lower losses and much longer plasmon lifetimes compared with conventional noble metals, and the spectral range where graphene plasmons can be excited-covering from microwave to optical frequencies-is significantly wider than the range covered by metallic plasmons. This has implications for a wide range of applications, including environmental management, detection of biological and chemical agents, and non-invasive medical diagnostics.

Motivated by the convenient plasmonic characteristics of graphene and the continuing advances in the fabrication of infrared active optoelectronic materials [14], a variety of structures with different geometries has been proposed in the literature. For instance, in [15], the authors studied the plasmon amplification properties on a plane spaser formed by two dense monolayers of nanocrystal quantum dots deposited onto both sides of a graphene nanoribbon. The structure exploits the spectral tunability via electrostatic gating and the high charge carrier mobility in graphene to amplify the low loss surface plasmons in a wide frequency band and with a relatively low threshold. Regarding spherical geometries, in Ref. [16], a spaser formed by a graphene nano-sphere wrapped with two-level quantum dots was theoretically studied. In particular, the authors evaluate the ability of the system to launch surface plasmons on a flat graphene sheet placed close to the sphere. In Ref. [17], the authors propose a graphene-metal hybrid plasmonic system 
surrounded by a quantum dots cascade stack as a nano spaser in the infrared. In addition, in Ref. [18], the authors study, within a full quantum approach in the electrostatic regime, a spaser design consisting of a semiconducting cylindrical wire wrapped by graphene with an active inner core given by a single quantum wire. Complete literature reviews of the major developments and latest advances in spaser theory, together with a systematic exposition of some of the key results useful for understanding the operation of spasers involving both bulk as well as $2 \mathrm{D}$ plasmonic materials, can be found in Refs. [5] (covering up to the year 2016) and [6].

This paper studies the non-radiative transfer of energy from the active medium to LSPs in a not-so-explored configuration, where a non-magnetic active medium in the form of a circular wire is coated with a monoatomic layer of graphene that separates the wire from an external and passive dielectric medium. We consider two different kind of wire cores: non-dispersive dielectric intrinsically non-plasmonic cores, and Drude dispersive plasmonic cores. In the first case, we assume that the core is made of an infrared and $\mathrm{THz}$ transparent material. Thus, in this case, the graphene layer introduces LSPs, which otherwise were absent in the bare wire. In the second case, we assume that the core is made of a metal-like material, such as a semiconductorbased nanocrystal [19-21] capable of supporting LSPs for wavelengths between $3 \mu \mathrm{m}$ and $10 \mu \mathrm{m}$. Thus, hybridization between graphene plasmons and those already existing in the bare wire occurs in this case.

Based on the resonant behavior of the spaser structure, the LSP characteristics on a graphene cylindrical spaser can be approached in two different but complementary ways. The first one, named the eigenmode approach, involves the study of the solutions to the boundary value problem without external sources [22], while the second one, the scattering approach, involves the study of the electromagnetic response of the structure when it is excited by an external source [23]. The first approach provides the dispersive characteristics of LSPs supported by the structure, such as dispersion curves and damping rates, and the second approach is related to the electromagnetic response via quantification of scattering observables when LSPs are externally excited. In a first stage, we use the eigenmode approach [22], particularly well-suited for finding the criticalgain values $\left[\operatorname{Im} \varepsilon_{1}\right]_{c}$ of the imaginary part of the permittivity of the active medium where the spaser condition is fulfilled. In the context of the eigenmode approach, these critical-gain values can be rigorously obtained by requiring simply that the imaginary part of a modal frequency be zero [24-26]. To do so, we use fully retarded methods in all of the examples presented here. Besides, we also invoke the quasistatic approximation, valid in the long wavelength limit, which allows us to obtain analytical expressions that explain the main results obtained with the rigorous theory.

In a second stage, we use the scattering approach, which provides a complementary understanding of plasmonic losses compensation and lasing conditions in terms of scattering observables such as scattering, extinction, and absorption cross sections.

This paper is organized as follows. In Section 2, we present a brief description of the electromagnetic theory based on separation of variables to obtain both the dispersion equation and the electromagnetic fields associated to LSPs supported by the structure, and we develop the quasistatic approximation. In Section 3, we present numerical examples for dielectric and metal-like wires covered with a graphene sheet. Finally, concluding remarks are provided in Section 4. The system of units used is Gaussian, and the $\exp (-i \omega t)$ time dependence is implicit throughout the paper, with $\omega$ the angular frequency, $t$ the time, and $i=\sqrt{-1}$. The symbols Re and Im are used for denoting the real and imaginary parts of a complex quantity, respectively.

\section{THEORY}

We consider a graphene-coated cylinder with circular cross section (radius $R$ ) centered at $x=0, y=0$ (see Fig. 1) embedded in a transparent medium with real valued electric permittivity $\varepsilon_{2}$ and magnetic permeability $\mu_{2}$. The active core is assumed to have a magnetic permeability $\mu_{1}=1$ and a complex valued electric permittivity $\varepsilon_{1}=\operatorname{Re} \varepsilon_{1}+i \operatorname{Im} \varepsilon_{1}$, with $\operatorname{Re} \varepsilon_{1}>0$ and $\operatorname{Im} \varepsilon_{1}<0$. Under these conditions, the gain coefficient of the core is $\beta_{g}=-k_{0} \operatorname{Im} \varepsilon_{1} / \sqrt{\operatorname{Re} \varepsilon_{1}}$ [27], where $k_{0}=\omega / c$ is the free space propagation constant. The graphene layer is treated as an infinitesimally thin, local, and isotropic two-sided layer with complex valued surface conductivity $\sigma(\omega)$, and we assume that $\mathrm{R}$ is large enough, so the constitutive properties of the graphene coating are the same as those of planar graphene. In this way, we can write $\sigma(\omega)=\sigma^{\text {intra }}(\omega)+\sigma^{\text {inter }}(\omega)$, with intraband $\left(\sigma^{\text {intra }}\right)$ and interband $\left(\sigma^{\text {inter }}\right)$ transition contributions given by the high-frequency expression derived from the Kubo formula [Eq. (1), Ref. [28]]:

$$
\begin{aligned}
\sigma^{\text {intra }}(\omega)= & \frac{2 i e^{2} k_{B} T}{\pi \hbar^{2}\left(\hbar \omega+i \gamma_{c}\right)} \ln \left[2 \cosh \left(\mu_{c} / 2 k_{B} T\right)\right], \\
\sigma^{\text {inter }}(\omega)= & \frac{e^{2}}{\hbar}\left\{\frac{1}{2}+\frac{1}{\pi} \arctan \left[\left(\hbar \omega-2 \mu_{c}\right) / 2 k_{B} T\right]\right. \\
& \left.-\frac{i}{2 \pi} \ln \left[\frac{\left(\hbar \omega+2 \mu_{c}\right)^{2}}{\left(\hbar \omega-2 \mu_{c}\right)^{2}+\left(2 k_{B} T\right)^{2}}\right]\right\},
\end{aligned}
$$

where $\mu_{c}$ is the chemical potential (controlled with the help of a gate voltage), $T$ is the ambient temperature, $\gamma_{c}$ is the carrier scattering rate, $k_{B}$ is the Boltzmann constant, $\hbar$ is the reduced Planck constant, and $e$ is the elementary charge. For large doping, $\mu_{c} \ll k_{B} T$, the intraband contribution of Eq. (1) dominates and takes the form predicted by the Drude model, whereas the interband contribution of Eq. (2) dominates for large frequencies $\hbar \omega>\mu_{c}[28,29]$.

\section{A. Rigorous Solution}

In order to derive the modal characteristics of the LSPs in the graphene-coated circular cross-section wire in terms of wire size, constitutive parameters of the substrate and ambient media, and the parameters of the graphene surface conductivity, we use an accurate electrodynamic approach that closely follows the approach of the usual Lorenz-Mie solution for geometries, where the radial and angular dependences of the fields can be separated. Thus, the electromagnetic field of LSPs in this case can be represented in terms of cylindrical multipole partial 


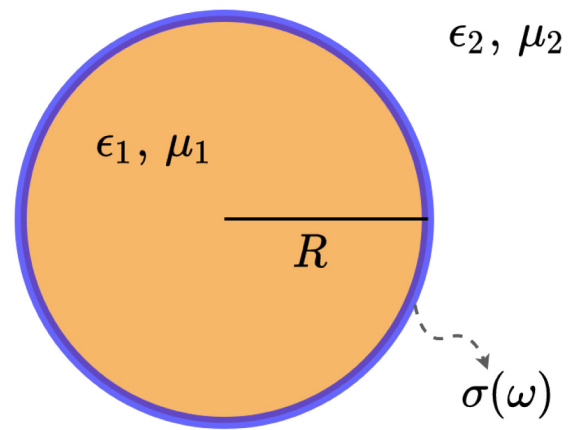

Fig. 1. Cylindrical spaser consisting of an active wire coated with a graphene monolayer immersed in an optically transparent medium.

waves characterized by discrete frequencies. For surface plasmons localized around the cylinder section, the problem can be handled in a scalar way, since LSPs are only supported in $p$ polarization, that is, when the electric field is parallel to the main section of the wire [22,23], and thus can induce the graphene coating electric currents along the azimuthal direction $\hat{\varphi}$.

The magnetic field $\vec{H}_{n}(\rho, \varphi, t)$ corresponding to the $n$th LSP mode is written as

$$
\vec{H}_{n}(\rho, \varphi, t)=F_{n}(\rho, \varphi) \exp \left(-i \omega_{n} t\right) \hat{z},
$$

where $\omega_{n}$ is the complex valued modal frequency. Due to carrier relaxation and radiation losses, plasmon oscillations in passive dissipative media are always damped, and thus the relation

$$
\operatorname{Im} \omega_{n}<0
$$

must be satisfied. Note that this relation holds even when $\operatorname{Im} \varepsilon_{1}=0$, that is, when the wire interior is a completely transparent dielectric medium. This is due to the losses given by the emission of radiation, which are always present. To find frequencies $\omega_{n}$ and field distributions $F_{n}$ associated with the $n$th LSP mode, $F_{n}(\rho, \varphi)$ is expanded as series of cylindrical harmonics in the internal and external regions:

$$
F_{n}(\rho, \varphi)=\left\{\begin{array}{cc}
c_{n} J_{n}\left(k_{1} \rho\right) \exp \operatorname{in} \varphi, & \rho<\mathrm{R}, \\
a_{n} H_{n}^{(1)}\left(k_{2} \rho\right) \exp \operatorname{in} \varphi, & \rho>\mathrm{R}
\end{array}\right.
$$

where $a_{n}$ and $c_{n}$ are complex coefficients, $n=1,2, \ldots \infty$, $k_{j}=\frac{\omega}{c} \sqrt{\varepsilon_{j} \mu_{j}}(j=1,2), c$ is the speed of light in vacuum, and $J_{n}$ and $H_{n}^{(1)}$ are the $n$th Bessel and Hankel functions of the first kind, respectively. Using the boundary conditions at the graphene layer $(\rho=R)$, we get a system of two homogeneous equations for the complex coefficients $a_{n}$ and $c_{n}$, and, by requiring that the determinant of this system of equations to be null, we obtain the following dispersion relation for the LSP eigenmodes represented by the cylindrical multipole partial wave in Eq. (3):

$$
\mu_{2} h_{n}-\mu_{1} j_{n}+i \mu_{1} \mu_{2} \frac{4 \pi}{c^{2}} \sigma \omega_{n} R j_{n} h_{n}=0
$$

where $j_{n}$ and $h_{n}$ are

$$
j_{n}=\frac{J_{n}^{\prime}\left(k_{1} R\right)}{k_{1} R J_{n}\left(k_{1} R\right)}, \quad h_{n}=\frac{H_{n}^{\prime(1)}\left(k_{2} R\right)}{k_{2} R H_{n}^{(1)}\left(k_{2} R\right)} .
$$

The prime denotes the first derivative with respect to the argument of the function. The eigenfrequency value fixes the relation between amplitudes of the inner and outer regions $\left(c_{n}\right.$ and $a_{n}$, respectively). Thus, the spatial part of the electromagnetic field for the $n$th mode can be written as

$$
\begin{gathered}
\vec{H}_{n}=\left\{\begin{array}{c}
J_{n}\left(k_{1} \rho\right) \exp \operatorname{in} \varphi \hat{z}, \quad \rho<\mathrm{R}, \\
\frac{k_{1} \varepsilon_{2}}{k_{2} \varepsilon_{1}} \frac{J_{n}^{\prime}\left(k_{1} R\right)}{H_{n}^{(1)}\left(k_{2} R\right)} H_{n}^{(1)}\left(k_{2} \rho\right) \exp \operatorname{in} \varphi \hat{z}, \quad \rho>\mathrm{R},
\end{array}\right. \\
\vec{E}_{n}=\left\{\begin{array}{c}
\frac{i c k_{1}}{\omega \varepsilon_{1}}\left(\operatorname{in} \frac{J_{n}\left(k_{1} \rho\right)}{k_{1} \rho} \hat{\rho}-J_{n}^{\prime}\left(k_{1} \rho\right) \hat{\varphi}\right) \exp \operatorname{in} \varphi, \quad \rho<\mathrm{R}, \\
i c k_{1} \frac{J_{n}^{\prime}\left(k_{1} R\right)}{\left.\omega \varepsilon_{1}\right)}\left(\operatorname{in} \frac{H_{n}^{(1)}\left(k_{2} \rho\right)}{H_{n}^{\prime 1}\left(k_{2} R\right)} \hat{\rho}-H_{n}^{\prime(1)}\left(k_{2} \rho\right) \hat{\varphi}\right) \exp \operatorname{in} \varphi, \quad \rho>\mathrm{R} .
\end{array}\right.
\end{gathered}
$$

\section{B. Gain Thresholds}

The optically active medium relies on the stimulated emission of radiation, and that depends, in turn, on the population inversion between an excited and a ground state of the active components of the medium, dyes, quantum dots, rare earth elements, etc. The saturation effects occur when the electromagnetic fields are so intense that a complete population inversion can no longer be sustained by the pumping mechanism, whatever it comes from (radiative or not radiative). Including saturation effects basically involves solving self-consistent rate equations, which typically consider the population of excited and ground states of the active material, the spatial distribution of electromagnetic fields, spontaneous emission (Purcell-enhanced or not), and the rate of stimulated emission. As discussed in several references $[30,31]$, the consequence of not taking into account saturation effects is that fields go to infinity once optical losses are exactly compensated. This is not a problem in principle if one is only interested in finding lasing conditions and not the intensity of the electromagnetic fields. Indeed, this property can be used for finding gain thresholds as divergences of properties such as the scattering coefficient [26,31]. Instead, here, we use another strategy, which is to find the critical value of the imaginary part of $\varepsilon_{1}$, for which the modal eigenfrequency $\omega_{n}$ is real [24-26].

Despite its limitations, the strategy used in the present work is still useful to correctly predict the mode that will be lasing (or spasing) and the frequency at which this occurs. Moreover, it can help to estimate the minimum gain required to observe an important enhancement of near and far fields, see, for example, Refs. [25,30]. The gain-loss compensation condition discussed in our work, also referenced as the gain threshold[26], does not necessarily mean that most emitted photons are the result of stimulated (coherent) emission, since, as calculations including saturation show, spontaneous (incoherent) emission can still be very important (especially if Purcell effects are relevant in the system studied). Requiring that most emitted photons come from spontaneous emission is a more strict condition that demands larger gain values than the ones informed here [32,33].

The permittivity of the active medium $\varepsilon_{1}$ is a frequencydependent property in general. However, when the response of the system is approximately the same for the frequency interval of interest, the wideband approximation can safely be used. This approximation, which implies taking $\varepsilon_{1}$ simply as a complex number, is useful for a first exploration of the system, as it allows 
one to calculate the optical response of a system independently of the characteristics of the active medium. As shown, for example, in Ref. [26], under the appropriate conditions, this approximation provides reliable results for the gain thresholds.

\section{Quasistatic Approximation}

When the size of the cylinder is small compared to the wavelength, $R \ll \lambda=2 \pi c / \omega$, we can use the quasistatic approximation. Using the small argument asymptotic expansions for Bessel and Hankel functions, the dispersion of Eq. (6) is written as [22]

$$
\varepsilon_{1}+\varepsilon_{2}=-\frac{4 \pi}{\omega} \sigma(\omega) \frac{i}{R} n .
$$

Equation (10) allows us to obtain analytic expressions for the LSP frequencies of the two cases: the non-dispersive and dispersive interiors. For large doping $\left(\mu_{c} \gg k_{B} T\right)$ and relatively low frequencies $\left(\hbar \omega \ll \mu_{c}\right)$, the intraband contribution of Eq. (1) to the surface conductivity plays the leading role. In this case, complex roots of Eq. (10) admit analytic expressions that can be obtained as follows.

\section{Non-Dispersive Medium}

By substituting the intraband term of Eq. (1) into Eq. (10), an analytical expression for the eigenfrequency for the non-dispersive case is obtained:

$$
\omega_{n}=\sqrt{\frac{\omega_{\mathrm{on}}^{2}}{\varepsilon_{1}+\varepsilon_{2}}-\left(\frac{\gamma_{c}}{2}\right)^{2}}-i \frac{\gamma_{c}}{2} \approx \frac{\omega_{\mathrm{on}}}{\sqrt{\varepsilon_{1}+\varepsilon_{2}}}-i \frac{\gamma_{c}}{2},
$$

where $\omega_{\text {on }}^{2}=\frac{4 e^{2} \mu_{c} n}{\hbar^{2} R}$ is the effective plasma frequency of the graphene coating for the $n$th mode. By replacing $\varepsilon_{1}=\operatorname{Re} \varepsilon_{1}+i \operatorname{Im} \varepsilon_{1}$ into Eq. (11) and taking into account that $x=\operatorname{Im} \varepsilon_{1} /\left(\operatorname{Re} \varepsilon_{1}+\varepsilon_{2}\right) \ll 1$, we can expand $\omega_{n}$ at the first order in $x$,

$$
\omega_{n} \approx \frac{\omega_{\mathrm{on}}}{\sqrt{\operatorname{Re} \varepsilon_{1}+\varepsilon_{2}}}-i \frac{1}{2}\left(\gamma_{c}+\frac{\omega_{\mathrm{on}} \operatorname{Im} \varepsilon_{1}}{\left[\operatorname{Re} \varepsilon_{1}+\varepsilon_{2}\right]^{3 / 2}}\right) .
$$

Note that, within this approximation, the real part of the eigenfrequency does not depend on the imaginary part of $\varepsilon_{1}$. In addition, from Eq. (12), we see that the critical value of the imaginary part of $\varepsilon_{1}$, for which the modal eigenfrequency is real, is written as

$$
\left[\operatorname{Im} \varepsilon_{1}\right]_{c}=-\frac{\left[\operatorname{Re} \varepsilon_{1}+\varepsilon_{2}\right]^{3 / 2} \gamma_{c}}{\omega_{\mathrm{on}}}=-\frac{\left[\operatorname{Re} \varepsilon_{1}+\varepsilon_{2}\right]^{3 / 2} \gamma_{c} \hbar R}{\sqrt{4 e^{2} \mu_{c} n}} .
$$

\section{Dispersive Medium}

We consider a mix of nanocrystals and dye (active medium) for the interior medium. For the metal-like behavior of the nanocrystal, we used the Drude model:

$$
\varepsilon_{\mathrm{DL}}(\omega)=\varepsilon_{\infty}-\frac{\omega_{p}^{2}}{\omega^{2}+i \gamma_{m} \omega},
$$

where $\varepsilon_{\infty}$ is the residual high-frequency response of the material, $\omega_{p}$ is the metallic plasma frequency, and $\gamma_{m}$ is the optical loss rate of the Drude material. Therefore, the effective homogenized permittivity of the medium inside the cylinder is

$$
\begin{aligned}
\varepsilon_{1}(\omega) & =\varepsilon_{\infty}-\frac{\omega_{p}^{2}}{\omega^{2}+i \gamma_{m} \omega}+\underbrace{\varepsilon_{\mathrm{dr}}+i \varepsilon_{\mathrm{di}}}_{\text {dye }} \\
& =\varepsilon_{\infty}^{\prime}-\frac{\omega_{p}^{2}}{\omega^{2}+i \gamma_{m} \omega}+i \varepsilon_{\mathrm{di}},
\end{aligned}
$$

where $\varepsilon_{\infty}^{\prime} \equiv \varepsilon_{\infty}+\varepsilon_{\mathrm{dr}}$ and $\varepsilon_{\mathrm{dr}}+i \varepsilon_{\mathrm{di}}$ represents the contribution of the dye to the effective homogenized permittivity. Note that in this mixed model of the active medium only the dye is taken in a wideband approximation.

Replacing in Eq. (10) the expression of $\varepsilon_{1}$ given by Eq. (15), after expanding in powers of $y=\frac{\varepsilon_{\mathrm{di}}}{\varepsilon_{\infty}^{\prime}+\varepsilon_{2}}$, we obtain

$\omega_{n}=\sqrt{\frac{\omega_{p}^{2}+\omega_{0 n}^{2}}{\varepsilon_{\infty}^{\prime}+\varepsilon_{2}}}-\frac{i}{2} \times\left[\frac{\omega_{p}^{2} \gamma_{m}+\omega_{0 n}^{2} \gamma_{c}}{\left(\omega_{p}^{2}+\omega_{0 n}^{2}\right)}+\varepsilon_{\mathrm{di}} \frac{\sqrt{\omega_{p}^{2}+\omega_{0 n}^{2}}}{\left(\varepsilon_{\infty}^{\prime}+\varepsilon_{2}\right)^{3 / 2}}\right]$.

Similar to the non-dispersive case, the real part of the modal eigenfrequencies does not depend on $\varepsilon_{\mathrm{di}}$ within the range of validity of the quasistatic approximation. Moreover, by equating to zero the imaginary part in Eq. (16), we obtain the critical value for $\varepsilon_{\mathrm{di}}$,

$$
\left[\varepsilon_{\mathrm{di}}\right]_{c}=-\frac{\omega_{p}^{2} \gamma_{m}+\omega_{0 n}^{2} \gamma_{c}}{\left(\omega_{p}^{2}+\omega_{0 n}^{2}\right)^{3 / 2}}\left(\varepsilon_{\infty}^{\prime}+\varepsilon_{2}\right)^{3 / 2},
$$

for which the lasing condition for the $n$th mode is reached.

\section{RESULTS}

In this section, we solve numerically the fully retarded dispersion relation of Eq. (6) to obtain both the complex eigenfrequencies and the gain thresholds for the first four multipolar modes. The calculation of the gain thresholds requires us to find the critical values for which the imaginary part of the eigenfrequency is zero, that is, the values that exactly compensate the plasmon losses. To do so, we minimize the modulus of Eq. (6) with respect to two variables, namely, the real part of the modal frequency and the optical gain. We use the Nelder-Mead optimization algorithm, taking the values provided by the quasistatic analytical expressions as initial guesses. We have considered that the wire is immersed in vacuum, $\varepsilon_{2}=\mu_{2}=1$, and that the graphene parameters are $\gamma_{c}=0.1 \mathrm{meV}$ and $\mathrm{T}=300 \mathrm{~K}$ in all of the calculations. Regarding the values of the constitutive parameters of the interior medium, and taking into account that our main purpose is to demonstrate spaser characteristics—such as gain thresholds and tunability-of graphene-coated active wires but not to reproduce the exact behavior of a given material, in the non-dispersive case, we have chosen values that are representative of Si- or Ge-based transparent dielectric materials, whereas in the dispersive case we have chosen constitutive parameter values that can be attained using semiconductor plasmonic nanocrystals [19-21]. For the case of dielectrics, the optical activity can be obtained by doping them with rare earth 


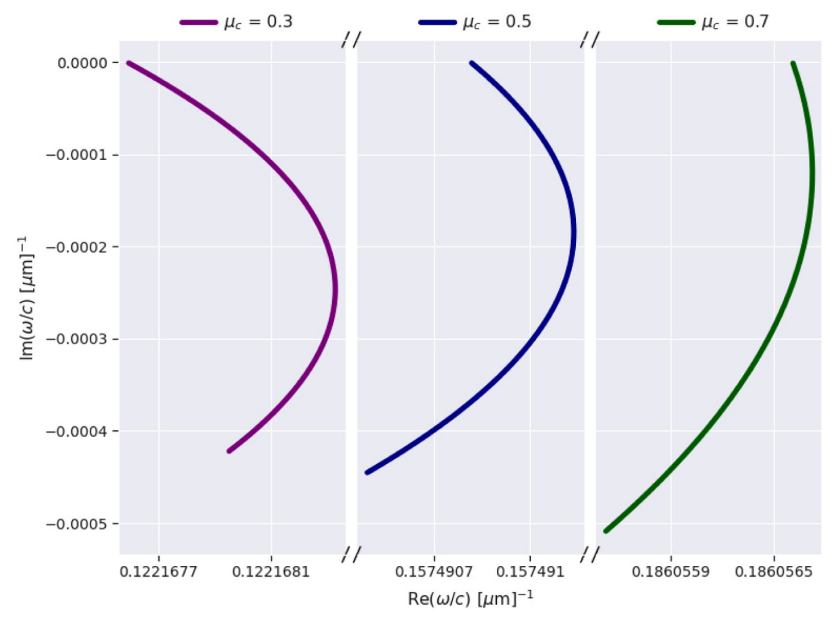

Fig. 2. Parametric curves of the complex poles (dipolar mode) for a cylinder with $\mathrm{R}=0.5 \mu \mathrm{m}, \operatorname{Re}\left(\varepsilon_{1}\right)=4.90, \hbar_{\gamma_{c}}=10^{-4} \mathrm{eV}, \mu_{1}=1$, $\mu_{2}=1, \varepsilon_{2}=1, \mathrm{~T}=300 \mathrm{~K}$, and three values of $\mu_{c}$.

ions [34] or organic dye molecules [2], while semiconductor nanocrystals can be pumped either electrically or optically [35].

\section{A. Non-Dispersive Medium}

We consider a dielectric wire of radius $R=0.5 \mu \mathrm{m}$ and $\operatorname{Re} \varepsilon_{1}=4$.9. In Fig. 2, we plotted the numerical solutions of the dispersion relation, Eq. (6), for the dipolar mode and for three different values of chemical potential $\mu_{c}=[0.3,0.6,0.9] \mathrm{eV}$. The solutions are displayed parametrically in the complex plane $\operatorname{Re} \omega / c-\operatorname{Im} \omega / c$, with the imaginary part of $\varepsilon_{1}$ as the parameter. The lowest part of the curves in Fig. 2 corresponds to a passive medium $\left(\operatorname{Im} \varepsilon_{1}=0\right)$. These curves approach the real axis when the value of $\operatorname{Im} \varepsilon_{1}$ approaches the critical value $\left[\operatorname{Im} \varepsilon_{1}\right]_{c}$, for which the lasing condition for the dipolar order is reached. We have verified that the curves cross the real axis when $\left|\operatorname{Im} \varepsilon_{1}\right|>\left|\operatorname{Im} \varepsilon_{1}\right|_{c}$.

The curves in Fig. 2 clearly show that, for the values of $\operatorname{Im} \varepsilon_{1}$ considered, the real part of the modal frequency remains almost constant. This behavior can be understood from the quasistatic expression in Eq. (12), where the real part of the eigenfrequency does not depend on the imaginary part of $\varepsilon_{1}$. In addition, we observe that the curves move towards higher $\operatorname{Re} \omega / c$ values when the value of the chemical potential is increased, a fact that blueshifts the lasing frequency. This behavior is also predicted by the quasistatic expression in Eq. (12), which shows that $\operatorname{Re} \omega_{n}$ behaves like $\sqrt{\mu_{c}}$.

Figure 3 shows the critical values $\left[\operatorname{Im} \varepsilon_{1}\right]_{c}$, for which the lasing condition is reached as a function of the chemical potential of graphene for the dipolar mode [Fig. 3(a)] and for quadrupolar, hexapolar, and octupolar modes [Fig. 3(b)].

From these curves, we see that the critical-gain coefficient $\beta_{g}=-k_{0} \operatorname{Im} \varepsilon_{1} / \operatorname{Re} \varepsilon_{1}$, for which the dipolar mode reaches the gain threshold, is greater than that corresponding to the other modes. This is due to the fact that radiation losses for the dipolar mode are higher than for other modes (see [22], Table 1), a behavior not predicted by the quasistatic approximation of Eq. (11). On the other hand, Eq. (13) anticipates that in the quasistatic approximation the critical-gain parameter behaves like $\mu^{-1 / 2}$. We observe that, while the numerical curves for

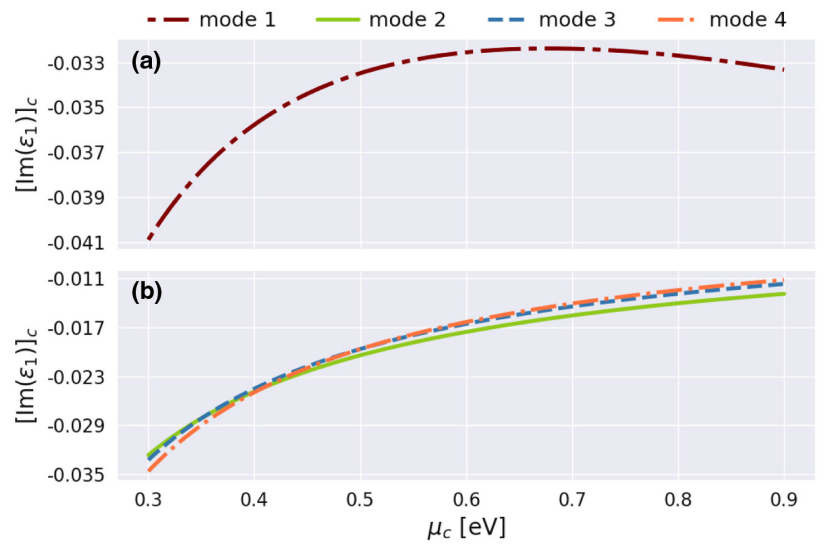

Fig. 3. Critical values of $\operatorname{Im} \varepsilon_{1}$ as a function of $\mu_{c}$ for a cylinder with $\mathrm{R}=0.5 \mu \mathrm{m}, \operatorname{Re}\left(\varepsilon_{1}\right)=4.90, \hbar_{\gamma_{c}}=10^{-4} \mathrm{eV}, \mu_{1}=1, \mu_{2}=1, \varepsilon_{2}=1$, and $\mathrm{T}=300 \mathrm{~K}$. (a) Dipolar and (b) the quadrupolar, hexapolar, and octupolar modes.

the higher (quadrupolar, hexapolar, and octupolar) modes in Fig. 3(b) exhibit this behavior, the numerical curve for the dipolar mode in Fig. 3(a) does not. This is in accordance with the fact that the higher the multipole modal frequency $\omega_{n}$, the better the quasistatic approximation, since the effective wavelength of higher multipoles becomes shorter, and the LSP modes perceive the circular graphene sheet as increasingly flat [see Ref. [22], Eqs. (11)-(13)].

In order to discuss the previous results in terms of scattering observables, in Figs. 4(a)-4(c), we plot color maps in the $\omega / c-\operatorname{Im} \varepsilon_{1}$ plane of the scattering, extinction, and absorption cross sections, respectively. Figure 4(a) illustrates the enhancement of the scattering efficiency for frequencies and gain parameters near the values $\omega_{c} / c=0.134$ and $\left[\operatorname{Im} \varepsilon_{1}\right]_{c}=-0.0353072$, for which the lasing condition for the dipolar mode is reached. Near this critical condition, the scattering cross section diverges, and the width at half-maximum of the resonance tends to zero, in agreement with the fact that the eigenfrequency tends to be real.

Figure 4(c) shows two regions separated by a green curve, for which the extinction cross section is equal to zero. Above this curve, the extinction cross section is positive (red region), indicating that plasmonic losses (ohmic more radiative losses) are not compensated, while below this curve the extinction cross section is negative, indicating that plasmonic losses are fully compensated. The critical point $\omega_{c} / c$ and $\left[\operatorname{Im} \varepsilon_{1}\right]_{c}$ falls on the full loss compensation curve, since the lasing condition implies the full loss compensation condition [3]. From Fig. 4(b), we see that the region for which the absorption cross section is positive falls above the full loss compensation curve, indicating that ohmic losses are compensated for an optical-gain coefficient value, which is lower than that corresponding to the critical value. This is an indication of deviations from the quasistatic approximation, which does not present radiation losses.

To gain insight about the active medium inclusion on the electromagnetic field scattered by the wire, in Fig. 5, we plotted the spatial distribution of the magnetic field $H_{z}$, at the resonance frequency for the first four modes (dipolar, quadrupolar, hexapolar, and octupolar), near the wire with an active medium $\left(\operatorname{Im} \varepsilon_{1} \neq 0\right.$, left column) and without an active 


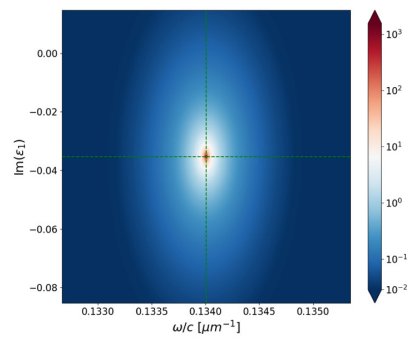

(a) Scattering



(b) Absorption

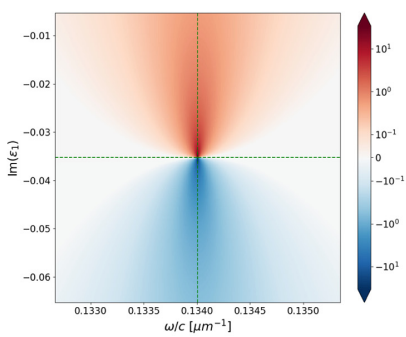

(c) Extinction

Fig. 4. Cross section for a cylinder with $\mathrm{R}=0.5 \mu \mathrm{m}$, $\operatorname{Re}\left(\varepsilon_{1}\right)=3.90, \mu_{c}=0.3 \mathrm{eV}, \hbar_{\gamma_{c}}=10^{-4} \mathrm{eV}, \mu_{1}=1, \mu_{2}=1, \varepsilon_{2}=1$, and $\mathrm{T}=300 \mathrm{~K}$. In green dotted lines: the critical values found before (Fig. 3).

medium ( $\operatorname{Im} \varepsilon_{1}=0$, right column). The direction of the plane wave incidence is from left to right. Each modal field has been normalized with respect to its own maximum field.

In Fig. 5, for the active medium, we used the critical values $\left[\operatorname{Im} \varepsilon_{1}\right]_{c}=$ $-0.0353072,-0.0271857,-0.0282580,-0.0298547$ for the imaginary part of the permittivity of the active medium and $\omega_{c} / c=[0.134005,0.189642,0.232135,0.267846] \mu^{-1}$ for the dipolar, quadrupolar, hexapolar, and octupolar modes.

By comparing the left and right columns of Fig. 5, obtained by evaluating the field distribution at each resonant frequency, we observe important differences when the active medium takes a gain value close to the critical one, $\left[\operatorname{Im} \varepsilon_{1}\right]_{c}$. These differences come from the interference with the incident plane wave. Basically, for gains far from the critical value, there is always interference between the fields produced by a given mode and the external source. However, close to the gain threshold, the fields produced by the eigenmodes are so strong that those coming from the external illumination become negligible.

\section{B. Dispersive Medium}

We present rigorous numerical results obtained when the cylinder core is a metal-like material, with dielectric permittivity $\varepsilon_{1}(\omega)$ described by Eq. (15). We use $\varepsilon_{\infty}=3.9$, plasma frequency $\hbar \omega_{p}=0.6 \mathrm{eV}$, and collision frequency $\hbar \gamma_{m}=0.01 \mathrm{eV}$.

In Fig. 6, we show parametric curves in the complex frequency plane of the dipolar eigenfrequency calculated by solving the dispersion relation of Eq. (6), as a parametric function of $\varepsilon_{\mathrm{di}}$ for three different values of chemical potential $\mu_{c}=[0.3,0.6,0.9] \mathrm{eV}$. As in the non-dispersive case, a very small variation in the real part of the eigenfrequency is observed, which is consistent with the fact that the expression for the real part of the eigenfrequency predicted by the quasistatic

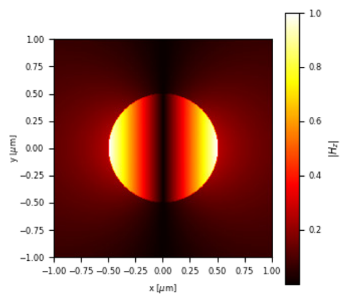

(a) mode $1, \operatorname{Im} \varepsilon_{1} \neq 0$

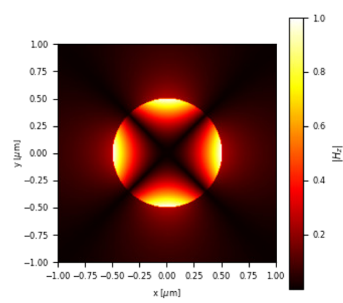

(c) mode $2, \operatorname{Im} \varepsilon_{1} \neq 0$

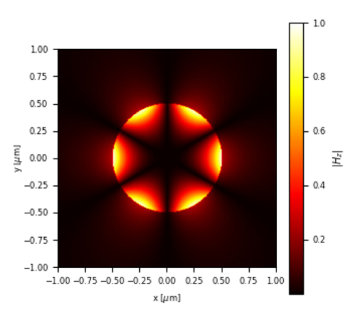

(e) mode $3, \operatorname{Im} \varepsilon_{1} \neq 0$

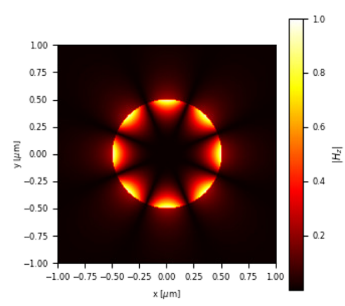

(g) mode $4, \operatorname{Im} \varepsilon_{1} \neq 0$

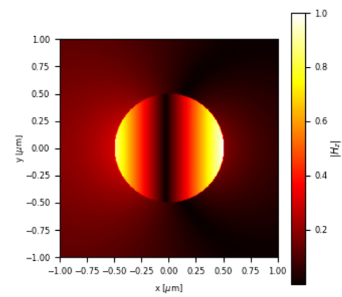

(b) mode $1, \operatorname{Im} \varepsilon_{1}=0$

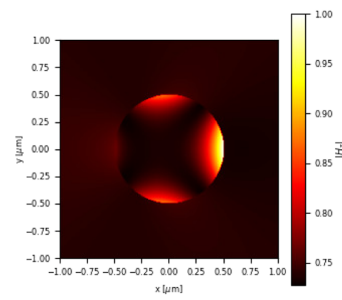

(d) mode $2, \operatorname{Im} \varepsilon_{1}=0$

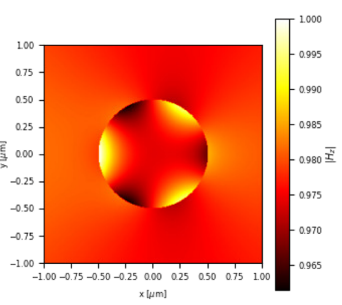

(f) mode $3, \operatorname{Im} \varepsilon_{1}=0$

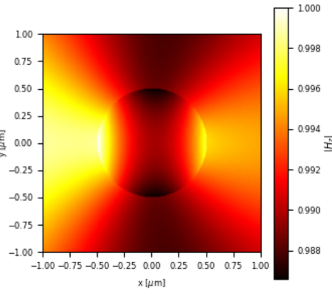

(h) mode $4, \operatorname{Im} \varepsilon_{1}=0$
Fig. 5. $|H z|$ field with $\mathrm{R}=0.5 \mu \mathrm{m}, \operatorname{Re}\left(\varepsilon_{1}\right)=3.90, \mu_{c}=0.3 \mathrm{eV}$, $\hbar_{\gamma_{c}}=10^{-4} \mathrm{eV}, \mu_{1}=1, \mu_{2}=1, \varepsilon_{2}=1$, and $\mathrm{T}=300 \mathrm{~K}$.

approximation of Eq. (16) is independent of $\varepsilon_{\mathrm{di}}$. However, the variation in the real part of the eigenfrequency is greater here than in the non-dispersive case (Fig. 2), which is in agreement with the fact that obtaining Eq. (16) involves the use of approximations (in particular expanding in powers of $y=\frac{\varepsilon_{\mathrm{di}}}{\varepsilon^{\prime} \infty+\varepsilon_{2}}$ ) that are not invoked to obtain Eq. (12). Moreover, we observe that the frequency region for the eigenfrequency trajectory is blue shifted when $\mu_{c}$ is increased from $0.3 \mathrm{eV}$ to $0.7 \mathrm{eV}$. This is consistent with the fact that the hybridization formula of Eq. (16), obtained from the quasistatic approximation, predicts a resonance frequency (real part of the eigenfrequency) that is proportional to $\sqrt{\omega_{p}^{2}+k \mu_{c}}, k=4 e^{2} /\left(\hbar^{2} R\right)$.

Figure 7 shows the critical values $\left[\varepsilon_{\mathrm{di}}\right]_{c}$ for the first four modes as a function of chemical potential $\mu_{c}$ for two wires sizes: $R=0.5 \mu \mathrm{m}$ [Fig. 7(a)] and $R=0.05 \mu \mathrm{m}$ [Fig. 7(b)]. Unlike the non-dispersive case, where the active medium only has to compensate for plasmon losses in the graphene monolayer, in metal-like dispersive cores, the active medium has to compensate for plasmon losses both in the graphene layer and in the nanocrystal. Thus, the modulus of the critical values $\left[\varepsilon_{\mathrm{di}}\right]_{c}$ for all 


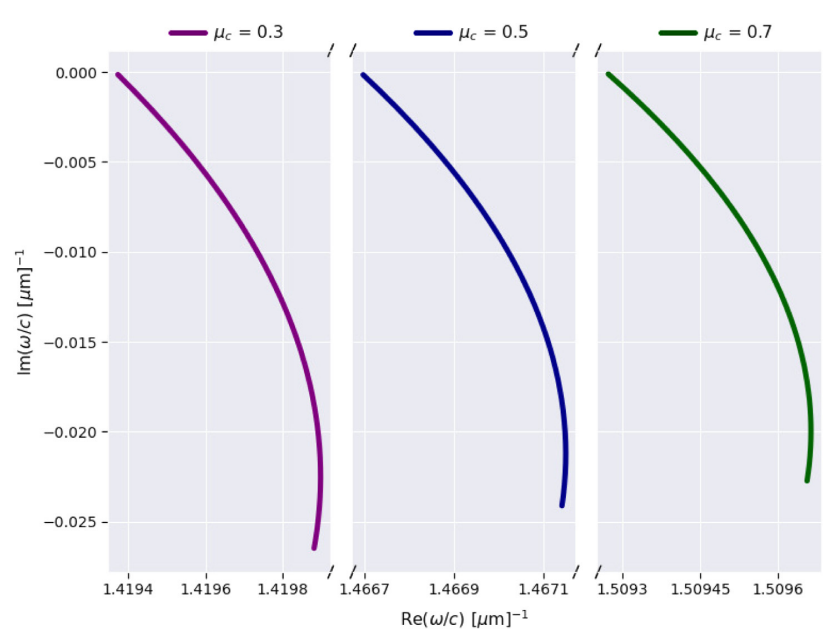

Fig. 6. Complex poles (dipolar mode) for a cylinder with $\mathrm{R}=0.5 \mu \mathrm{m}, \varepsilon_{\infty}=3.9, E_{p}=0.6 \mathrm{eV}, \hbar_{\gamma_{m}}=0.01 \mathrm{eV}, \hbar_{\gamma_{c}}=10^{-4} \mathrm{eV}$, $\mu_{1}=1, \mu_{2}=1, \varepsilon_{2}=1$, and $\mathrm{T}=300 \mathrm{~K}$.



(a) $\mathrm{R}=0.5 \mu \mathrm{m}$

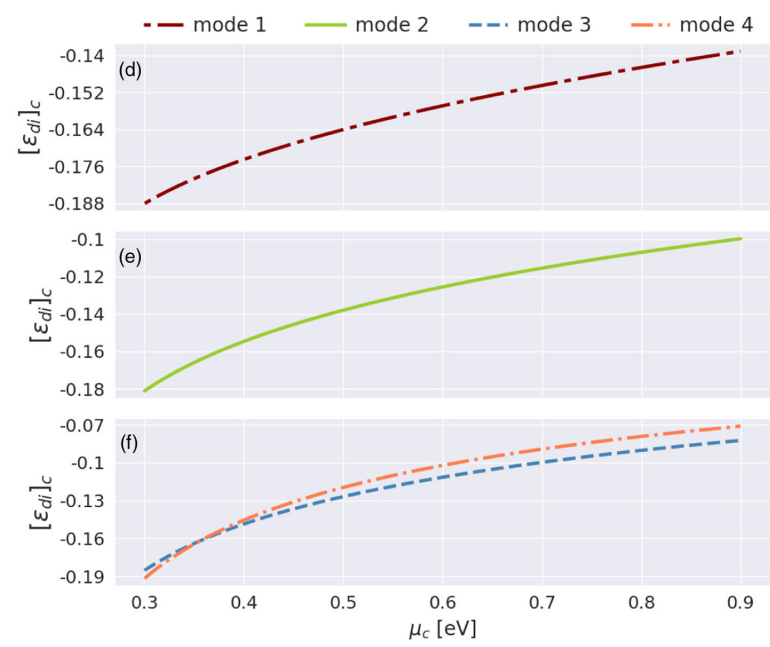

(b) $\mathrm{R}=0.05 \mu \mathrm{m}$

Fig. 7. Critical values of $\varepsilon_{\mathrm{di}}$ as a function of $\mu$ for a cylinder with $\varepsilon_{\infty}=3.9, \quad E_{p}=0.6 \mathrm{eV}, \quad \hbar_{\gamma_{m}}=0.01 \mathrm{eV}, \quad \hbar_{\gamma_{c}}=10^{-4} \mathrm{eV}, \quad \mu_{1}=1$, $\mu_{2}=1, \varepsilon_{2}=1$, and $\mathrm{T}=300 \mathrm{~K}$. the graphene-nanocrystal hybridized plasmon modes shown in Fig. 7 are bigger than those for the graphene plasmons obtained in the non-dispersive case (Fig. 3).

Regarding wire size, Fig. 7 clearly shows that the critical values corresponding to $R=0.05 \mu \mathrm{m}$ [Fig. 7(b)] are notably lower than those corresponding to $R=0.5 \mu \mathrm{m}$ [Fig. 7(a)]. This reduction of the value of the critical gain with particle size is anticipated by the quasistatic expression of Eq. (17), which shows that $\left|\left[\varepsilon_{\mathrm{di}}\right]_{c}\right|$ is an increasing function of $R$ (note that the dependence on $R$ is included in $\omega_{0 n}^{2}$ ). It is interesting to note that this behavior, which is absent in metallic cylinders without a graphene cover, results from the graphene-nanocrystal plasmon hybridization and is highlighted when the effective plasma frequency $\omega_{\text {on }}$ is comparable with the nanocrystal plasma frequency $\omega_{p}$ (as is the case for the constitutive parameters chosen in this example).

In Fig. 8, we plot color maps, in the $\omega / c-\operatorname{Im} \varepsilon_{1}$ plane, of the scattering [Fig. 8(a)], extinction [Fig. 8(b)], and absorption [Fig. 8(c)] cross sections. We observe that the behavior around the critical point $\left(\omega_{c} / c,\left[\varepsilon_{\mathrm{di}}\right]_{c}\right)$ is similar to that observed in the non-dispersive case (Fig. 4), where the critical point falls on the full loss compensation curve that separates the passive region [red region in Fig. 8(c)] from the active region [blue region in Fig. 8(c)].

In Fig. 9, we give color maps of the spatial distribution of the magnetic field for the first four modes. The right column corresponds to the case of a core without gain $\left(\varepsilon_{\mathrm{di}}=0\right)$, whereas the left column corresponds to the case of an active core $\left(\varepsilon_{\mathrm{di}} \neq 0\right)$. The values of $\varepsilon_{\mathrm{di}} \neq 0$ have been taken to obtain almost full loss compensation for each mode. We used the critical values $\left[\varepsilon_{\mathrm{di}}\right]_{c}=-0.253806,-0.203944,-0.183008,-0.174493$ for the imaginary part of the permittivity of the active medium, and, for the frequencies, we used the values $\omega_{c} / c=[0.798031,0.894243,0.977434,1.05174] \mu \mathrm{m}^{-1}$ for the dipolar, quadrupolar, hexapolar, and octupolar modes.

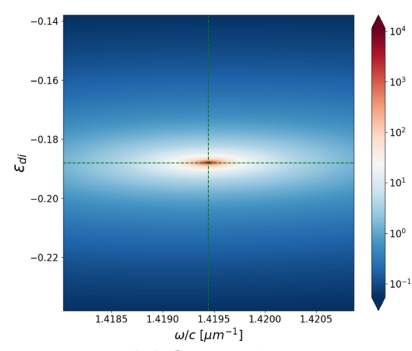

(a) Scattering

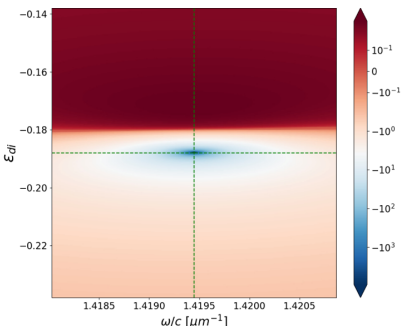

(b) Absorption

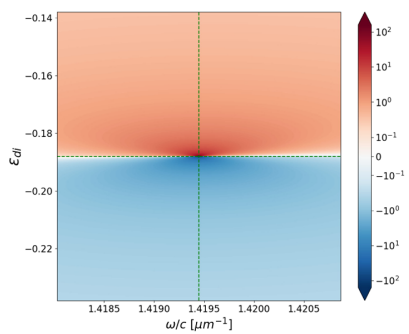

(c) Extinction

Fig. 8. Cross section for a cylinder with $\mathrm{R}=0.05 \mu \mathrm{m}, \varepsilon_{\infty}=3.9$, $E_{p}=0.6 \mathrm{eV}, \quad \hbar_{\gamma_{m}}=0.01 \mathrm{eV}, \quad \mu_{c}=0.3003 \mathrm{eV}, \quad \hbar_{\gamma_{c}}=10^{-4} \mathrm{eV}$, $\mu_{1}=1, \mu_{2}=1, \varepsilon_{2}=1$, and $\mathrm{T}=300 \mathrm{~K}$. In green dotted lines: the critical values found before. 




(a) mode $1, \varepsilon_{d i} \neq 0$

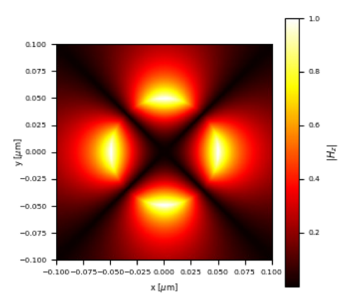

(c) mode 2, $\varepsilon_{d i} \neq 0$

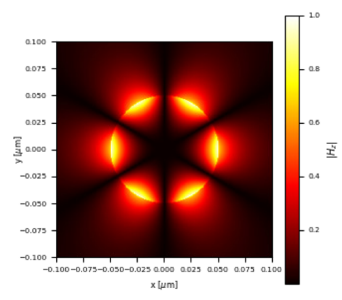

(e) mode $3, \varepsilon_{d i} \neq 0$

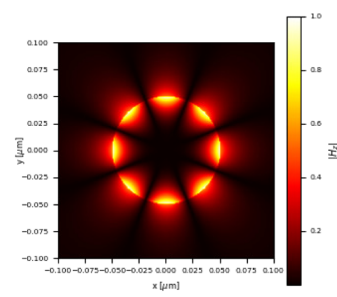

(g) mode $4, \varepsilon_{d i} \neq 0$

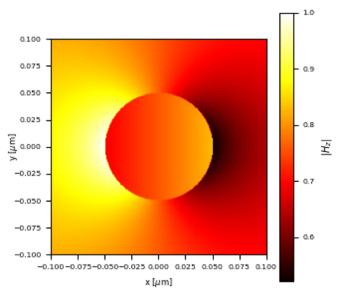

(b) mode $1, \varepsilon_{d i}=0$
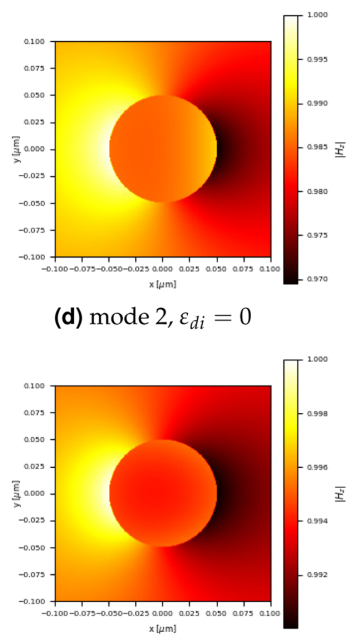

(f) mode $3, \varepsilon_{d i}=0$

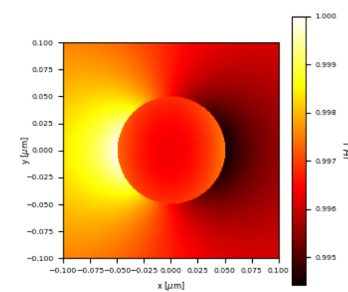

(h) mode $4, \varepsilon_{d i}=0$ (d) mode 2, $\varepsilon_{d i}=0$

Fig. 9. $|H z|$ field with $\mathrm{R}=0.05 \mu \mathrm{m}, \varepsilon_{\infty}=3.9, E_{p}=0.6 \mathrm{eV}$, $\hbar_{\gamma_{m}}=0.01 \mathrm{eV}, \mu_{c}=0.3 \mathrm{eV}, \hbar_{\gamma_{c}}=10^{-4} \mathrm{eV}, \mu_{1}=1, \mu_{2}=1, \varepsilon_{2}=1$, and $\mathrm{T}=300 \mathrm{~K}$.

We again observe that the inclusion of gain inside the cylinder sharply highlights the multipolar characteristics of the near field.

\section{CONCLUSION}

We have investigated the lasing and optical amplification conditions for the LSP modes on a cylindrical wire wrapped with graphene. Regarding the material of the cylindrical wire, two different cases have been considered: an infrared/THz transparent material and a nanocrystal (a metal-like material). While in the first case the active medium compensates plasmon losses only in the graphene monolayer, in the second case, the active medium compensates losses both in the graphene layer and in the nanocrystal.

In a first stage, we used an eigenmode approach to calculate the trajectories of the eigenfrequencies in the complex frequency plane when the optical-gain parameter is varied. This procedure allowed us to obtain the critical values of the optical gain for which a modal eigenfrequency trajectory crosses the real axis. It is for these critical values that the lasing condition is fulfilled for that mode. In a second stage, we used a scattering approach, which allowed us to get a complementary understanding of plasmonic losses compensation and lasing conditions in terms of scattering observables such as scattering, extinction, and absorption cross sections. To analyze the results, we invoke analytical expressions obtained by using the quasistatic approximation. Our findings show that the studied systems present a wide frequency range tunability of lasing resonant states. Moreover, the gain modal critical values exhibit a great dependence on chemical potential. Wires with smaller radius show much smaller gain modal critical values. Of the studied modes, the dipolar one showed the largest gain modal critical values. Both results suggest that radiative losses are the key factor controlling the gain modal critical values.

We believe that these results provide a deeper understanding of the characteristics of LSP spasers based on graphene and will motivate further exploration of other spaser configurations exploiting the optical advantages of the graphene electromagnetic response in the infrared and $\mathrm{THz}$ ranges. This may find numerous applications in $\mathrm{THz}$ spectroscopy, $\mathrm{THz}$ imaging, or in sensing of biological samples, for example, where tissues are typically transparent to the frequency range studied.

Funding. Secretaria de Ciencia y Tecnología-Universidad Nacional de Córdoba ; Consejo Nacional de Investigaciones Científicas y Técnicas; Agencia Nacional de Promoción Científica y Tecnológica (PICT-2018-03587).

Acknowledgment. We acknowledge financial support by Consejo Nacional de Investigaciones Científicas y Técnicas (CONICET); Secretaría de Ciencia y Tecnología de la Universidad Nacional de Córdoba (SECYT-UNC); and Agencia Nacional de Promoción Científica y Tecnológica.

Disclosures. The authors declare no conflicts of interest.

Data Availability. Data underlying the results presented in this paper are not publicly available at this time but may be obtained from the authors upon reasonable request.

\section{REFERENCES}

1. D. J. Bergman and M. I. Stockman, "Surface plasmon amplification by stimulated emission of radiation: quantum generation of coherent surface plasmons in nanosystems," Phys. Rev. Lett. 90, 027402 (2003).

2. M. Noginov, G. Zhu, A. Belgrave, R. Bakker, V. Shalaev, E. Narimanov, S. Stout, E. Herz, T. Suteewong, and U. Wiesner, "Demonstration of a spaser-based nanolaser," Nature 460, 1110-1112 (2009).

3. M. I. Stockman, "Spasers explained," Nat. Photonics 2, 327-329 (2008).

4. D. Wang, W. Wang, M. P. Knudson, G. C. Schatz, and T. W. Odom, "Structural engineering in plasmon nanolasers," Chem. Rev. 118, 2865-2881 (2017).

5. M. Premaratne and M. I. Stockman, "Theory and technology of spasers," Adv. Opt. Photon. 9, 79-128 (2017).

6. S. I. Azzam, A. V. Kildishev, R.-M. Ma, C.-Z. Ning, R. Oulton, V. M. Shalaev, M. I. Stockman, J.-L. Xu, and X. Zhang, "Ten years of spasers and plasmonic nanolasers," Light Sci. Appl. 9, 90 (2020).

7. N. Zheludev, S. Prosvirnin, N. Papasimakis, and V. A. Fedotov, "Lasing spaser," Nature Photonics 2, 351-354 (2008).

8. M. Moccia, G. Castaldi, A. Alu, and V. Galdi, "Harnessing spectral singularities in non- Hermitian cylindrical structures," IEEE Trans. Antennas Propag. 68, 1704-1716 (2020).

9. B. Liu, W. Zhu, S. D. Gunapala, M. I. Stockman, and M. Premaratne, "Open resonator electric spaser," ACS Nano 11, 12573-12582 (2017).

10. G.-M. Pan, D.-J. Yang, L. Zhou, Z.-H. Hao, and Q.-Q. Wang, "Enhanced second harmonic generation by mode matching in gain-assisted double-plasmonic resonance nanostructure," Sci. Rep. 7, 9776 (2017). 
11. G.-M. Pan, D.-J. Yang, L. Zhou, and Z.-H. Hao, "Low-loss resonance modes in a gain-assisted plasmonic multimer," J. Phys. D 51, 115104 (2018).

12. J.-H. Wang, Z. Gao, P. Song, P. N. Melentiev, X.-H. Wang, H. Gao, F. Yang, B. Kang, J.-J. Xu, and H.-Y. Chen, "How gain layer design determines performance of nanoparticle-based spaser," J. Phys. Chem. C 124, 16553-16560 (2020).

13. A. Krasnok and A. Alù, "Active nanophotonics," Proc. IEEE 108, 628-654 (2020).

14. H. Lu, G. M. Carroll, N. R. Neale, and M. C. Beard, "Infrared quantum dots: progress, challenges, and opportunities," ACS Nano 13, 939-953 (2019).

15. O. L. Berman, R. Y. Kezerashvili, and Y. E. Lozovik, "Graphene nanoribbon based spaser," Phys. Rev. B 88, 235424 (2013).

16. S. B. Ardakani and R. Faez, "Tunable spherical graphene surface plasmon amplification by stimulated emission of radiation," J. Nanophoton. 13, 026009 (2019).

17. M. M. Tohari, A. Lyras, and M. S. AlSalhi, "A novel metal nanoparticles-graphene nanodisks-quantum dots hybrid-systembased spaser," Nanomaterials 10, 416 (2020).

18. S. B. Ardakani and R. Faez, "Spaser based on graphene tube," Sci. Iran. D 27, 3084-3095 (2020).

19. P. West, S. Ishii, G. Naik, N. Emani, V. Shalaev, and A. Boltasseva, "Searching for better plasmonic materials," Laser Photon. Rev. 4, 795-808 (2010).

20. Z. Liu, Y. Zhong, I. Shafei, S. Jeong, L. Wang, H. T. Nguyen, C. J. Sun, T. Li, J. Chen, L. Chen, Y. Losovyj, X. Gao, W. Ma, and X. Ye, "Broadband tunable mid-infrared plasmon resonances in cadmium oxide nanocrystals induced by size-dependent nonstoichiometry," Nano Lett. 20, 2821-2828 (2020).

21. L. Wang, M. H. Kafshgari, and M. Meunier, "Optical properties and applications of plasmonic-metal nanoparticles," Adv. Funct. Mater. 30, 2005400 (2020).

22. M. Riso, M. Cuevas, and R. A. Depine, "Complex frequencies and field distributions of localized surface plasmon modes in graphene coated subwavelength wires," J. Quant. Spectrosc. Radiat. Transfer 173, 26-33 (2016).

23. M. Riso, M. Cuevas, and R. A. Depine, "Tunable plasmonic enhancement of light scattering and absorption in graphene coated subwavelength wires," J. Opt. 17, 075001 (2015).
24. E. I. Smotrova, V. O. Byelobrov, T. M. Benson, J. Čtyroký, R. Sauleau, and A. I. Nosich, "Optical theorem helps understand thresholds of lasing in microcavities with active regions," IEEE J. Quantum Electron. 47, 20-30 (2011).

25. M. Natarov, T. M. Benson, and A. I. Nosich, "Electromagnetic analysis of the lasing thresholds of hybrid plasmon modes of a silver tube nanolaser with active core and active shell," Beilstein J. Nanotechnol. 10, 294-304 (2019).

26. N. Passarelli, R. Bustos-Marún, and R. Depine, "Lasing conditions of transverse electromagnetic modes in metallic-coated micro- and nanotubes," J. Phys. Chem. C 123, 13015-13026 (2019).

27. S. A. Maier, "Gain-assisted propagation of electromagnetic energy in subwavelength surface plasmon polariton gap waveguides," Opt. Commun. 258, 295-299 (2006).

28. L. A. Falkovsky, "Optical properties of graphene and IV-VI semiconductors," Phys. Usp. 51, 887-897 (2008).

29. R. A. Depine, Graphene Optics: Electromagnetic Solution of Canonical Problems (Morgan and Claypool, 2017).

30. N. Arnold, K. Piglmayer, A. V. Kildishev, and T. A. Klar, "Spasers with retardation and gain saturation: electrodynamic description of fields and optical cross-sections," Opt. Mater. Express 5, 2546-2577 (2015).

31. N. Passarelli, R. A. Bustos-Marún, and E. A. Coronado, "Spaser and optical amplification conditions in gold-coated active nanoparticles," J. Phys. Chem. C 120, 24941-24949 (2016).

32. J. B. Khurgin and G. Sun, "Injection pumped single mode surface plasmon generators: threshold, linewidth, and coherence," Opt. Express 20, 15309-15325 (2012).

33. J. B. Khurgin and M. A. Noginov, "How do the Purcell factor, the Q-factor, and the beta factor affect the laser threshold?" Laser Photon. Rev. 15, 2000250 (2021).

34. P. Molina, E. Yraola, M. O. Ramírez, C. Tserkezis, J. L. Plaza, J. Aizpurua, J. Bravo-Abad, and L. E. Bausá, "Plasmon-assisted $\mathrm{Nd}^{3+}$-based solid-state nanolaser," Nano Lett. 16, 895-899 (2016).

35. R. A. Flynn, C. S. Kim, I. Vurgaftman, M. Kim, J. R. Meyer, A. Mäkinen, K. Bussmann, L. Cheng, F.-S. Choa, and J. Long, "A roomtemperature semiconductor spaser operating near $1.5 \mu \mathrm{m}$," Opt. Express 19, 8954-8961 (2011). 